\title{
SINGULARITY PROFILE IN THE MEAN CURVATURE FLOW*
}

\author{
WEIMIN SHENG ${ }^{\dagger}$ AND XU-JIA WANG ${ }^{\ddagger}$
}

\begin{abstract}
In this paper we study the geometry of first time singularities of the mean curvature flow. By the curvature pinching estimate of Huisken and Sinestrari, we prove that a mean curvature flow of hypersurfaces in the Euclidean space $\mathbb{R}^{n+1}$ with positive mean curvature is $\kappa$-noncollapsing, and a blow-up sequence converges locally smoothly along a subsequence to a smooth, convex blow-up solution. As a consequence we obtain a local Harnack inequality for the mean convex flow.
\end{abstract}

Key words. Mean curvature flow, singularity profile, $\kappa$-noncollapsing.

AMS subject classifications. 53C44, 35K55

1. Introduction. In this paper we are concerned with the geometry of the first time singularities of the mean curvature flow of closed, smooth hypersurfaces in the Euclidean space $\mathbb{R}^{n+1}$. The singularity profile has been studied by many authors $[5$, $8-13,15,21,22]$, but in general it can be extremely complicated. For example an open problem is whether for any $k>1$, there is a self-similar toric solution of genus $k$ in $\mathbb{R}^{3}$. Therefore as in $[8-13,21,22]$, we restrict to the mean curvature flow of hypersurfaces with positive mean curvature, namely the mean convex flow.

For the mean convex flow, Huisken and Sinestrari [11, 12] proved the following crucial one-side curvature pinching estimate, namely at any point up to the first time singularity,

$$
\lambda_{1} \geq-\varphi\left(\lambda_{1}+\cdots+\lambda_{n}\right),
$$

where $\lambda_{1} \leq \cdots \leq \lambda_{n}$ are the principal curvatures and $\varphi$ is a nonnegative function depending on the initial hypersurface and satisfying $\varphi(t) / t \rightarrow 0$ as $t \rightarrow \infty$. This estimate corresponds to a one-side curvature pinching estimate of Hamilton and Ivey (see [6]) for the Ricci flow of 3-manifolds. By estimate (1.1) and Hamilton's Harnack inequality [7], Huisken and Sinestrari proved that the blow-up sequence at the maximal mean curvature points of type II singularities converges along a subsequence to a convex translating solution.

In this paper we study the asymptotic behavior of general blow-up sequences. First we prove (see $\S 3$ for terminology and notation).

TheOREM 1.1. Let $\mathcal{F}=\left\{F_{t}\right\}_{t \in[0, T)}$ be a smooth, mean convex flow of closed hypersurface in $\mathbb{R}^{n+1}, n \geq 2$, with first singular time $T$. Then any blow-up sequence $\mathcal{F}^{k}=\left\{F_{t}^{k}\right\}$ at the first time singularity sub-converges locally smoothly to a convex solution $\mathcal{F}^{\prime}=\left\{F_{t}^{\prime}\right\}_{t \in\left(-\infty, T^{\prime}\right)}$, where $0 \leq T^{\prime} \leq+\infty$.

The local smooth convergence means for any $R>0, \mathcal{F}^{k} \cap Q_{R}$ converges in the $C^{3}$ topology, where $Q_{R}$ denotes the parabolic cube in spacetime.

We then prove the $\kappa$-noncollapsing. By our definition in $\S 3$, the $\kappa$-noncollapsing means a pinching of the solution by a sphere from inside. Recall that (1.1) then means a pinching from outside.

\footnotetext{
${ }^{*}$ Received November 24, 2008; accepted for publication January 30, 2009.

${ }^{\dagger}$ Department of Mathematics, Zhejiang University, Hangzhou 310027, China (weimins@zju. edu.cn). The first author was supported by NSFC10771189 and 10831008.

${ }_{\ddagger}^{\ddagger}$ Centre for Mathematics and Its Applications, Australian National University, Canberra ACT 0200, Australia (Xu-Jia.Wang@anu.edu.au). The second author was supported by ARC DP0664517 and DP0879422.
} 
TheOREm 1.2. Let $\mathcal{F}=\left\{F_{t}\right\}_{t \in[0, T)}$ be a smooth, mean convex flow of closed hypersurface in $\mathbb{R}^{n+1}, n \geq 2$. Then $\mathcal{F}$ is $\kappa$-noncollapsing up to the first singular time $T$, for some constant $\kappa>0$ depending only on $n$ and the initial surface $F_{0}$.

Note that the $\kappa$-noncollapsing is invariant under dilation. Hence Theorem 1.2 implies a blow-up sequence at any points in the flow $\mathcal{F}$ is $\kappa$-noncollapsing with the same $\kappa$. By the convexity of blow-up solutions, we can improve Theorem 1.2 to

THEOREM 1.3. There exists a constant $\kappa^{*}>0$ depending only on $n$, such that any normalized limit flow to any given mean convex flow in $\mathbb{R}^{n+1}$ at the first time singularities is $\kappa^{*}$-noncollapsing.

The normalized condition in Theorem 1.3 is only to exclude hyperplane as limit flows. Theorem 1.3 can be applied to mean curvature flow with surgeries. To prove Theorem 1.2, we show that a pair of hyperplanes, including a multiplicity two plane, cannot be a blow-up solution. From Theorem 1.2, we have

COROLlaRY 1.1. The grim reaper is not blow-up solution.

Here the grim reaper is the translating solution determined by hypersurface $\{x \in$ $\left.\mathbb{R}^{n+1}: x_{n+1}=-\log \cos x_{1}\right\}$, which is the product of $\mathbb{R}^{n-1}$ with the curve $x_{n+1}=$ $-\log \cos x_{1}$ in a 2-plane.

COROLlary 1.2. The set of normalized blow-up solutions is compact.

By our definition in $\S 3$, the mean curvature of a normalized blow-up solution is equal to 1 at the origin in space time. Hence by Theorem 1.1, a hyperplane is not a normalized blow-up solution. If one includes hyperplanes as blow-up solutions (at regular points), then the set of all blow-up solutions whose mean curvature is uniformly bounded by a positive constant is compact.

From Theorems 1.1 and 1.2, we have a local Harnack inequality.

Corollary 1.3. Let $\mathcal{F}=\left\{F_{t}\right\}_{t \in[0, T)}$ be a mean convex flow. Then for any $R>0$, there exists $\delta>0$, depending only on $n, R$, and the initial condition $F_{0}$, such that for any point $p=\left(x_{0}, t_{0}\right) \in \mathcal{F}$ at which the mean curvature is greater than $\delta^{-1}$, and any point $q \in \mathcal{F}$ in the parabolic cube $Q_{R / H}(p)$, we have

$$
\delta H(q) \leq H(p) \leq \delta^{-1} H(q),
$$

where $H$ is the mean curvature at $p$.

Note that if one can prove the local Harnack inequality first, by the curvature pinching estimate (1.1), one obtains immediately Theorems 1.1 and 1.2.

Theorem 1.1 asserts that every blow-up solution is convex. But to understand the geometry of the singularities more precisely, we wish to classify all blow-up solutions, or more generally all ancient convex solutions, as a blow-up solution must be ancient. The study of ancient convex solutions was carried out by the second author in [20]. The following results have been obtained:

(a) A blow-up translating solution in $\mathbb{R}^{3}$ is rotationally symmetric.

(b) There exists non-rotationally symmetric translating solution in $\mathbb{R}^{n+1}$ for $n>2$.

(c) The blow-down of a blow-up solution is a shrinking sphere or cylinder.

(d) Let $\mathcal{F}=\left\{F_{t}\right\}_{t \in[0, T)}$ be a smooth, mean convex flow of closed surface in $\mathbb{R}^{3}$. Then at any point $x_{0} \in F_{t_{0}}$ with large mean curvature, the surface $F_{t_{0}}$ is, after normalization, very close to either a cylinder or a convex cap. 
Part (d) can be restated as follows: For any $\varepsilon>0, R>1$, there exists $r_{\varepsilon, R}>1$ such that for any normalized blow-up solution $\mathcal{F}^{\prime}=\left\{F_{t}^{\prime}\right\}$ in $\mathbb{R}^{3}$, there is a compact set $G \subset F_{0}^{\prime}$ with diameter $\leq r_{\varepsilon, R}$, such that for any $p \in F_{0}^{\prime} \backslash G$, the component $\mathbb{B}_{R / H}(p) \cap F_{0}^{\prime}$ is, after normalization, in the $\varepsilon$-neighborhood of the cylinder $S^{1} \times \mathbb{R}^{1}$, where $H$ is the mean curvature of $F_{0}^{\prime}$ at $p$.

The above results show that the singularity profile of mean convex flow is in line with those of Perelman for the Ricci flow of 3-manifolds [17]. In particular our results for mean convex flow in $\mathbb{R}^{3}$ correspond to those for the Ricci flow, therefore it is conceivable that one can carry out surgeries as in [13]. We will not get into details in this direction. We point out that the idea of our proof is different from that of Perelman. Perelman needs to prove the $\kappa$-noncollapsing and classify ancient $\kappa$-noncollapsing solutions before he obtains the local smooth convergence of blow-up sequences. We use the curvature pinching estimate (1.1) and some basic estimates of parabolic equations to prove the smooth convergence (Theorem 1.1) first, and then use it to obtain other results.

Some related results have been obtained in a recent paper [13], in which Huisken and Sinestrari studied the mean curvature flow with surgeries in $\mathbb{R}^{n+1}$. They proved that when $n \geq 3$ and the sum of the least two principal curvatures $\lambda_{1}$ and $\lambda_{2}$ is positive, a blow-up sequence sub-converges locally smoothly to a convex blow-up solution and every blow-up solution is very close to a canonical one.

In an earlier work [21] (\$12), prior to Perelman's paper [17], White proved that the grim reaper and a multiplicity two plane cannot be blow-up solutions, which was used in [22] to prove that a blow-up sequence sub-converges locally smoothly to a convex blow-up solution. Therefore Theorems 1.1 and 1.2 belong to White [21, 22]. His proof contains many novel ideas but nevertheless is very involved and difficult to understand.

Most recently, Ben Andrews discovered a direct geometric proof of the $\kappa$ noncollapsing, which, together with (1.1), also implies the smooth convergence to a convex solution in Theorem 1.1. There are many other interesting geometric flows in Euclidean space or Riemannian manifolds. Different methods may apply to different situations. Our argument may apply to curvature flows of hypersurfaces provided one can establish a similar one-side curvature pinching estimate as (1.1) and related interior a priori estimates.

This paper is arranged as follows. We include in $\S 2$ some preliminaries. In $\S 3$ we introduce the terminology and notation. In $\S 4$ we prove the convexity of limit flows. In $\S 5$ we prove that a semi-noncollapsing blow-up sequence converges locally smoothly to a limit flow. In $\S 6$ we prove any blow-up sequence is semi-noncollapsing. The proof of Theorems 1.1-1.3 is finished in $\S 7$. Finally in $\S 8$ we discuss tangent flows.

The authors would like to thank Shizhong Du and Oliver Schnürer for carefully reading this paper and their helpful comments. 
2. Preliminaries. A time-dependent, smooth embedding $F_{t}=F(\cdot, t): \mathcal{M} \rightarrow$ $\mathbb{R}^{n+1}$, where $t \in[0, T)$, is a solution to the mean curvature flow if

$$
\frac{\partial}{\partial t} F(p, t)=H \cdot \nu, \quad p \in \mathcal{M}, t \in[0, T),
$$

where $H$ is the mean curvature, $\nu$ is the unit inward normal. When $F_{t}(\mathcal{M})$ is locally a graph, we have the parabolic equation

$$
\frac{\partial u}{\partial t}=\sqrt{1+|D u|^{2}} \operatorname{div}\left(\frac{D u}{\sqrt{1+|D u|^{2}}}\right) .
$$

It is well known that the mean curvature $H$ satisfies the equation [8]

$$
\frac{\partial H}{\partial t}=\Delta H+H|A|^{2} .
$$

Since $F_{0}(\mathcal{M})$ is a closed hypersurface, it follows that if $H \geq 0$ at $t=0$, then $H>0$ at $t>0$. From (2.3) we also see that if $u$ is a solution of $(2.2)$ in $Q_{r}^{n}:=\mathbb{B}_{r}^{n}(0) \times\left[0, r^{2}\right]$ with $0 \leq H \leq C$, then we have the Harnack inequality $H\left(0, r^{2}\right) \geq C_{1} \sup H$, where the sup is taken in the domain $\mathbb{B}_{r / 2}^{n}(0) \times\left[\frac{1}{4} r^{2}, \frac{3}{4} r^{2}\right]$. In particular if $H\left(0, r^{2}\right)=0$, then $H \equiv 0$ in $Q_{r}^{n}$, and $u$ is a linear function by (2.4) below.

For a mean convex flow $\mathcal{F}$, we also have the estimate for the second fundamental form $A$,

$$
|A|^{2} \leq C H^{2}
$$

for some constant $C$ depending on $n$ and $F_{0}$. Indeed, (2.4) follows from the following equation [10],

$$
\frac{\partial}{\partial t}\left(\frac{|A|^{2}}{H^{2}}\right)=\Delta\left(\frac{|A|^{2}}{H^{2}}\right)+\frac{2}{H}\left\langle\nabla H, \nabla\left(\frac{|A|^{2}}{H^{2}}\right)\right\rangle-\frac{2}{H^{4}}\left|H \nabla_{i} h_{j k}-\nabla_{i} H \cdot h_{j k}\right|^{2} .
$$

For the mean curvature flow of graphs, we have the interior gradient and second derivative estimates. That is if $u$ is a smooth solution to $(2.2)$ in $Q_{r}^{n}$, then for $0<$ $t \leq r^{2}$,

$$
\begin{aligned}
& |D u|(0, t) \leq \exp \left(C_{1}+C_{2} M^{2}\left(\frac{1}{r^{2}}+\frac{1}{t}\right)\right) \\
& \left|D^{2} u\right|(0, t) \leq C_{3}\left(1+\sup _{Q_{r}^{n}}|D u|^{4}\left(\frac{1}{r^{2}}+\frac{1}{t}\right)\right),
\end{aligned}
$$

where $M=\sup _{Q_{r}^{n}}|u|, C_{1}, C_{2}$ depend only on $n$, and $C_{3}$ depends on $n$ and $M$.

Estimates (2.5) and (2.6) were established in [5]. The estimate (2.5) in [5] also depends on the Lipschitz continuity of the initial condition. But by choosing the auxiliary function

$$
G\left(x^{\prime}, t, \xi\right)=t \rho\left(x^{\prime}\right) \varphi(u) \log u_{\xi}\left(x^{\prime}, t\right)
$$

as in [19], where $x^{\prime}=\left(x_{1}, \cdots, x_{n}\right)$ (see notation in $\S 3$ ), $\rho\left(x^{\prime}\right)=1-\left|x^{\prime}\right|^{2} / r^{2}$ is a cut-off function, $\varphi(u)=1+u / M$, one obtains (2.5) by the computation in [19]. 
3. Terminology and notation. First we recall the terminology in [21]. Let $\mathcal{F}=\left\{F_{t}\right\}_{t \in[0, T)}$ be a mean convex flow, which develops first time singularity at time $T$. For any sequences $p_{k}=\left(x_{k}, t_{k}\right) \in \mathcal{F}$ and $a_{k} \rightarrow \infty$, let $\mathcal{F}^{k}=\mathcal{D}_{a_{k}, p_{k}}(\mathcal{F})$, where

$$
\mathcal{D}_{a_{k}, p_{k}}:(x, t) \rightarrow\left(a_{k}\left(x-x_{k}\right), a_{k}^{2}\left(t-t_{k}\right)\right)
$$

namely one first makes a translation such that $p_{k}=\left(x_{k}, t_{k}\right)$ becomes the origin in space-time, and then make a parabolic dilation of scale $a_{k}$. We get a blow-up sequence $\mathcal{F}^{k}$. If $a_{k}=H\left(x_{k}, t_{k}\right)$ is the mean curvature of $\mathcal{F}$ at $\left(x_{k}, t_{k}\right)$, we call $\mathcal{F}^{k}$ a normalized blow-up sequence at $\left(x_{k}, t_{k}\right)$. Note that for a normalized blow-up sequence, $\left(x_{k}, t_{k}\right)$ must converges to a singular point (due to the assumption $a_{k} \rightarrow \infty$ ).

If $\mathcal{F}^{k}$ converges locally smoothly (i.e. smoothly in any compact set) to a mean curvature flow $\mathcal{F}^{\prime}$, we say $\mathcal{F}^{\prime}$ is a limit flow, or a blow-up solution. If $\mathcal{F}^{k}$ is a normalized blow-up sequence, the limit flow (blow-up solution) is accordingly called normalized.

Tangent flow. A limit flow is called tangent flow if the sequence $\left(x_{k}, t_{k}\right)=\left(x_{0}, t_{0}\right)$ is a fixed point.

Ancient solution. A solution to the mean curvature flow is ancient if it exists from time $t=-\infty$.

Eternal solution. A solution to the mean curvature flow is eternal if it exists for time $t$ from $-\infty$ to $\infty$.

Collapsing. A blow-up sequence $\mathcal{F}^{k}$ is collapsing if there is no ball $\mathbb{B}_{r}\left(x_{0}\right)$ contained in $U_{t}^{k}$ (for some $x_{0}$ and any $t<0$ ) for infinitely many $k$ 's (see notation below).

Semi-noncollapsing. A blow-up sequence $\mathcal{F}^{k}$ is semi-noncollapsing if there is ball $\mathbb{B}_{r}\left(x_{0}\right)$ and a time $t_{0}$ such that $\mathbb{B}_{r}\left(x_{0}\right) \subset U_{t_{0}}^{k}$ for infinitely many $k$ 's.

$\kappa$-noncollapsing. For any point $(x, t) \in \mathcal{F}$, we say $\mathcal{F}$ is $\kappa$-noncollapsing at $(x, t) \in$ $\mathcal{F}$ if $r_{x, t} H(x, t) \geq \kappa$, where $\kappa>0$ is a constant, $H(x, t)$ is the mean curvature of $\mathcal{F}$ at $(x, t)$, and

$$
r_{x, t}=\sup \left\{\rho: \mathbb{B}_{\rho}(z) \subset U_{t} \text { and }|z-x|=\rho\right\}
$$

( $r_{x, t}$ is the radius of the largest ball which is contained in $U_{t}$ and tangential to $F_{t}$ at $x$ ). We say $\mathcal{F}$ is $\kappa$-noncollapsing if it is $\kappa$-noncollapsing at any point before the first time singularity.

The grim reaper: It is the translating solution given by $x_{n+1}=-\log \cos x_{1}$ (it is the product of a curve with $\mathbb{R}^{n-1}$ ).

REMARK 3.1.

(i) By $(3.1)$, the point $(0,0)$ always belongs to $\mathcal{F}^{k}$ for all $k$, and also belongs to the limit flow $\mathcal{F}^{\prime}$ if it exists. Moreover, the mean curvature of a normalized blow-up sequence $\mathcal{F}^{k}$ is equal to 1 at $(0,0)$.

(ii) A limit flow is an ancient flow.

(iii) The $\kappa$-noncollapsing is invariant under translation and dilation of coordinates. Hence if $\mathcal{F}$ is $\kappa$-noncollapsing, so is any blow-up sequence with the same $\kappa$.

(iv) We always have $\kappa \leq n$.

(v) It is easy to verify that $r_{x, t}$ depends continuously on $(x, t)$ and $\mathcal{F}$. Namely if $\mathcal{F}^{k}$ converges locally smoothly to $\mathcal{F}^{\prime}$, and $\mathcal{F}^{k} \ni\left(x_{k}, t_{k}\right) \rightarrow\left(x_{0}, t_{0}\right) \in \mathcal{F}$, then we have $r_{x_{k}, t_{k}}\left[\mathcal{F}^{k}\right] \rightarrow r_{x_{0}, t_{0}}\left[\mathcal{F}^{\prime}\right]$, where $r_{x_{k}, t_{k}}\left[\mathcal{F}^{k}\right]$ is the radius in (3.2) relative to $\mathcal{F}^{k}$.

(vi) A semi-noncollapsing blow-up sequence may contain a collapsing component, but we will rule out the case in $\S 5$ (Remark 5.1). Also a collapsing blow-up sequence may contain two or more collapsing components. 
Notation.

$\mathcal{M}:$ an oriented, compact $n$-dimensional differential manifold without boundary.

$\mathcal{F}=\left\{F_{t}\right\}_{t \in[0, T)}$ : smooth solution to the mean curvature flow with initial condition $F_{0}$, on a maximal time interval $[0, T)$ for some $T \in(0, \infty]$.

$F_{t}=F(\cdot, t)(0 \leq t<T): \quad$ a smooth embedding of $\mathcal{M}$ in $\mathbb{R}^{n+1}$.

$F_{t}$ : $\quad$ we also use $F_{t}$ to denote the hypersurface $F_{t}(\mathcal{M})$, and $F_{0}=F_{t \mid t=0}$.

$U_{t}: \quad$ the open domain enclosed by $F_{t}(\mathcal{M}), U_{0}=U_{t \mid t=0}$, and $\mathcal{U}=\left\{U_{t}\right\}_{t \in[0, T)}$.

$\bar{U}_{t}: \quad$ closure of $U_{t}, \bar{U}_{t}=U_{t} \cup \partial U_{t}$.

$\mathbb{B}_{r}(x)=\mathbb{B}_{r}^{(n+1)}(x): \quad$ the ball in $\mathbb{R}^{n+1}$ of radius $r$ with center at $x$.

$\mathbb{B}_{r}^{n}\left(x^{\prime}\right)$ : the ball in $\mathbb{R}^{n}$ of radius $r$ with center at $x^{\prime}$.

$Q_{r}\left(x_{0}, t_{0}\right): \quad$ the parabolic cube $\mathbb{B}_{r}\left(x_{0}\right) \times\left(t_{0}-r^{2}, t_{0}\right]$ in spacetime.

$|\cdot|_{\mathcal{H}^{k}}: \quad k$-dimensional Hausdorff measure.

For a blow-up sequence $\mathcal{F}^{k}=\left\{F_{t}^{k}\right\}$ (or a limit flow $\mathcal{F}^{\prime}=\left\{F_{t}^{\prime}\right\}$ ), we denote accordingly by $U_{t}^{k}$ (or $U_{t}^{\prime}$ ) the open set enclosed by $F_{t}^{k}$ (or $F_{t}^{\prime}$ ).

In this paper, we use $x=\left(x_{1}, x_{2} \cdots, x_{n+1}\right)$ to denote a point in $\mathbb{R}^{n+1}$, and use $x^{\prime}=\left(x_{1}, x_{2} \cdots, x_{n}\right)$ to denote a point in $\mathbb{R}^{n}$.

\section{Convexity of limit flow.}

LEMma 4.1. If a blow-up sequence $\mathcal{F}^{k}$ converges locally smoothly to a limit flow $\mathcal{F}^{\prime}$, then $\mathcal{F}^{\prime}$ is convex, namely $U_{t}^{\prime}$ is convex whenever it is nonempty.

Proof. For any time $t_{0}$ such that $U_{t_{0}}^{\prime}$ is not empty, it suffices to prove that for any two interior points $z_{0}, z_{1} \in U_{t_{0}}^{\prime}$, the line segment $\overline{z_{0} z_{1}}$ lies in $U_{t_{0}}^{\prime}$.

By a proper translation and rotation of coordinates, we assume that $z_{0}=$ $(0, \cdots, 0,1)$ and $z_{1}=(0, \cdots, 0,-1)$. Since $z_{0}, z_{1}$ are interior points of $U_{t_{0}}^{\prime}$, there exists small $\delta>0$ such that $\mathbb{B}_{4 \delta}\left(z_{0}\right), \mathbb{B}_{4 \delta}\left(z_{1}\right) \subset U_{t_{0}}^{\prime}$. Denote

$$
\Omega=\left\{x \in \mathbb{R}^{n+1}: \quad \sum_{i=1}^{n} x_{i}^{2}<\delta^{2}\left(1+x_{n+1}^{2}\right),-1<x_{n+1}<1\right\} .
$$

Since the initial hypersurface $F_{0}$ is smooth and mean convex, the singularity set is strictly contained in the domain enclosed by $F_{0}$. Hence $\Omega \subset U_{t}^{k}$ for any large $k$, provided $-t$ is sufficiently large. If the line segment $\bar{z}_{0} z_{1}$ is not contained in $U_{t_{0}}^{\prime}$, we decrease $t$ to a moment $\bar{t}=\bar{t}_{k}$ such that $\Omega \subset U_{\bar{t}}^{k}$ but $\partial \Omega$ contacts with the boundary of $U_{\bar{t}}^{k}$ at some point $z^{*}$, namely

$$
\bar{t}=\sup \left\{s: \Omega \subset U_{t}^{k} \forall t<s\right\} .
$$

Since $\mathbb{B}_{4 \delta}\left(z_{0}\right), \mathbb{B}_{4 \delta}\left(z_{1}\right) \subset U_{t_{0}}^{\prime}$, we have $z^{*} \in \partial \Omega \cap\left\{-1<x_{n+1}<1\right\}$. Let $\lambda$ and $\Lambda$ be the least and largest principal curvatures of $F_{\bar{t}}^{k}$ at this point. By comparing the principal curvatures of $\partial \Omega$ and $F_{t_{0}}^{k}$, we have $\lambda \leq-\frac{1}{4} \delta$ and $\Lambda \leq 4 \delta^{-1}$. Hence the mean curvature $H \leq C \delta^{-1}$ by (2.4). Applying these estimates to the curvature pinching (1.1) we obtain $-\delta a_{k} \geq-\varphi\left(C \delta^{-1} a_{k}\right)$. Note that $\delta>0$ is fixed, $a_{k} \rightarrow \infty$, and $\varphi(t) / t \rightarrow 0$ as $t \rightarrow \infty$, we reach a contradiction. $\square$

The above proof is inspired by an idea in [22] (pages 130-131, [22]). We made some changes to make use of the curvature pinching estimate (1.1). Note that the curvature pinching estimate in $[11,12]$ is written in a different way, but it is easy to see that it implies (1.1).

5. Smooth convergence. We prove a local curvature estimate (5.3), from which it follows the smooth convergence of a semi-noncollapsing blow-up sequence. 
Given constants $\delta, d \in(0,1]$, we say a mean convex flow $\mathcal{F}$ has property $P_{\delta, d}^{a}$ at a point $p_{0}=\left(x_{0}, t_{0}\right) \in \mathcal{F}$ if (a) below is satisfied; and $\mathcal{F}$ has property $P_{\delta, d}^{a, b}$ at $p_{0}$ if both (a) and (b) are satisfied; where

(a) $\exists y_{0} \in \mathbb{R}^{n+1}$ s.t. $\left|x_{0}-y_{0}\right|=d, \mathbb{B}_{\delta d}\left(y_{0}\right) \subset U_{t_{0}}$, and the line segment $x_{0} y_{0} \subset \bar{U}_{t_{0}}$;

(b) $F_{t} \cap \mathbb{B}_{10 d}\left(x_{0}\right) \subset N_{\delta d / 32}\left(\Gamma_{t}\right)$ for some convex hypersurface $\Gamma_{t}, \forall t \in\left(t_{0}-(\delta d)^{2}, t_{0}\right]$, where $N_{\delta}$ denotes the $\delta$-neighborhood.

Assume $\mathcal{F}$ has property $P_{\delta, d}^{a, b}$ at $p_{0}$. Choose a coordinate system such that $x_{0}$ is the origin and $y_{0}=(0, \cdots, 0, d)$. Then $\Gamma_{t} \cap\left\{\left|x^{\prime}\right|<\frac{\delta d}{2}\right\}$ is the graph of a Lipschitz continuous, convex function $\varphi(\cdot, t)$ defined in $\mathbb{B}_{\delta d / 2}^{n}(0), t \in\left(t_{0}-(\delta d)^{2}, t_{0}\right]$.

Claim: $\left|\varphi\left(x^{\prime}, t\right)\right| \leq 8 d$ for any $x^{\prime} \in \mathbb{B}_{\delta d / 2}^{n}(0)$ and $t \in\left(t_{0}-(\delta d / 8)^{2}, t_{0}\right]$.

Indeed, by assumption (a) we have $\varphi(\cdot, t) \leq d$ in $\mathbb{B}_{\delta d / 2}^{n}(0)$, for any $t \in\left(t_{0}-\right.$ $\left.\left(\frac{\delta d}{8}\right)^{2}, t_{0}\right]$. Since the origin $0 \in \mathcal{F}_{t_{0}}^{k}$, by the convexity of $\varphi$ we have $\varphi\left(\cdot, t_{0}\right) \geq-2 d$. For $t \in\left(t_{0}-\left(\frac{\delta d}{8}\right)^{2}, t_{0}\right]$, noting that $\mathcal{F}$ is a mean convex flow, by assumption (b) we see that up to a small perturbation, $\varphi(\cdot, t)$ is increasing in $t$. If $\varphi\left(\cdot, t_{0}-\left(\frac{\delta d}{8}\right)^{2}\right) \leq-8 d$ at some point in $\mathbb{B}_{\delta d / 2}^{n}(0)$, by convexity we have $\varphi\left(\cdot, t_{0}-\left(\frac{\delta d}{8}\right)^{2}\right) \leq-3 d$ in $\mathbb{B}_{\delta d / 4}^{n}(0)$. A simple application of the comparison principle for the mean curvature flow implies that $\varphi\left(0, t_{0}\right) \leq-d$, which is in contradiction with (b). The Claim is proved.

Suppose $F_{t} \cap \mathbb{B}_{\delta d / 2}(0)$ is also a graph of a function $u(\cdot, t)$ for $t \in\left(t_{0}-\left(\frac{\delta d}{8}\right)^{2}, t_{0}\right]$. By the interior gradient estimate (2.5), we have

$$
\left|D_{x^{\prime}} u\left(x^{\prime}, t\right)\right| \leq C_{\delta, n}^{\prime}
$$

for $\left|x^{\prime}\right|<\delta d / 4$ and $t \in\left(t_{0}-\left(\frac{\delta d}{16}\right)^{2}, t_{0}\right]$. (For application to a blow-up sequence below, we don't need to use the interior gradient estimate (2.5) to get (5.1). Indeed, by Lemma 5.1 and the smooth convergence in the proof of Lemma 5.2, the estimate (5.1) follows from the Lipschitz continuity of the convex function $\varphi$ ). Hence by the interior estimate (2.6), we have

$$
\Lambda_{\mathcal{F}}(0) \leq C_{\delta, n}^{\prime \prime} / d
$$

where $\Lambda_{\mathcal{F}}(p)=\left|\lambda_{1}(p)\right|+\cdots+\left|\lambda_{n}(p)\right|$, and $\lambda_{1}(p), \cdots, \lambda_{n}(p)$ are the principal curvatures of $F_{t}$ at $x, p=(x, t) \in \mathcal{F}$. The constants $C_{\delta, n}^{\prime}$ and $C_{\delta, n}^{\prime \prime}$ depend only on $\delta$ and $n$ but are independent of $d$, which can be easily seen by making the dilation $x \rightarrow x / d$.

Next we prove a technical lemma. Denote $\mathcal{C}_{x_{0}, \delta}=\left\{x \in \mathbb{R}^{n+1}: x=x_{0}+t(y-\right.$ $\left.\left.x_{0}\right), t \in(0,1), y \in \mathbb{B}_{\delta}(0)\right\}$ an open round cone with vertex at $x_{0}, x_{0} \notin \mathbb{B}_{\delta}(0)$.

Lemma 5.1. Let $U$ be an open set in $\mathbb{R}^{n+1}$ with smooth boundary $F$. Suppose $\mathbb{B}_{r}(0) \subset U \subset \mathbb{B}_{R}(0)$. Then $\exists \varepsilon>0$, depending only on $n, r, R$, such that the following conclusion holds: If at any point $x_{0} \in F$ with $\mathcal{C}_{x_{0}, r / 4} \subset U$, the principal curvatures of $F \geq-\varepsilon$, then $\mathcal{C}_{x_{0}, r / 2} \subset U$ for all $x_{0} \in F$.

Proof. If the lemma is not true, assume $\inf \left\{|x|: x \in F: \mathcal{C}_{x, r / 2} \not \subset U\right\}$ is attained at $x_{0}$. Let $P$ be the two-dimensional plane determined by $0, x_{0}$, and $\nu$, where $\nu$ is the normal of $F$ at $x_{0}$. Let $\ell$ be the intersection of $P$ with $F$. Then the tangent line of $\ell$ at $x_{0}$ is also tangential to $\partial \mathbb{B}_{r / 2}(0)$, and a tangent plane of $F$ at $x_{0}$ is also a tangent plane of $\partial \mathbb{B}_{r / 2}(0)$. Let us parameterize $\ell=\{x(s): s \geq 0\}$ by its arclength, starting at $x_{0}$, and let $\alpha(s)=|x(s)|$. Then when $s>0$ small, $\alpha$ is decreasing in $s$. Let $\left[0, s_{0}\right]$ be the maximal interval in which $\alpha(s) \leq \alpha(0)$. By our choice of $x_{0}$, we have $\mathcal{C}_{x(s), r / 2} \subset U$ for all $s \in\left(0, s_{0}\right]$. Hence by assumption, the principal curvatures of $F$ at $x(s) \geq-\varepsilon$. Since $\mathcal{C}_{x(s), r / 2} \subset U$, we have $\langle\nu(s),-x(s)\rangle \geq c_{0}>0$, where $\nu(s)$ is 
the normal of $F$ at $x(s)$. Hence the curvature of the curve $\ell>-C \varepsilon$. When $\varepsilon>0$ is sufficiently small, the curve $\ell$ must intersect with the ball $\mathbb{B}_{r}(0)$ (as the tangent line of $\ell$ at $x_{0}$ is tangential to $\partial \mathbb{B}_{r / 2}(0)$ ), which is in contradiction with the assumption $U \supset \mathbb{B}_{r}(0)$.

When the condition $U \subset \mathbb{B}_{R}(0)$ is dropped, we consider the set $U \cap \mathbb{B}_{R}(0)$. By approximation we have

Lemma 5.1'. Let $U$ be an open set in $\mathbb{R}^{n+1}$ with smooth boundary F. Suppose $\mathbb{B}_{r}(0) \subset U$. Then $\exists \varepsilon>0$, depending only on $n, r, R$, such that the following conclusion holds: If at any point $x_{0} \in F$ with $\left|x_{0}\right|<R$ and $\mathcal{C}_{x_{0}, r / 4} \subset U$, the principal curvatures of $F \geq-\varepsilon$, then $\mathcal{C}_{x_{0}, r / 2} \subset U$ for any $x_{0} \in F$ with $\left|x_{0}\right|<R$.

Lemma 5.1 (and 5.1') implies that for any given $R>0$, there is a component of $F \cap \mathbb{B}_{R}(0)$ which can be represented as a Lipschitz continuous radial graph over the unit sphere, and there is no other components inside this component. We call this component the noncollapsing component of $\mathcal{F}$.

Let $\mathcal{F}^{k}$ be a blow-up sequence. We fix a small $\delta>0$ such that $\mathcal{F}^{k}$ has the property $P_{\delta, d}^{a}$ at the origin for some $d \leq 1$. Note that for any smooth mean curvature flow $\mathcal{F}$ and any $p \in \mathcal{F}, \mathcal{F}$ has property $P_{\delta, d}^{a}$ at $p$ with $\delta \rightarrow 1$ as $d \rightarrow 0$. Hence for any $p \in \mathcal{F}^{k}$, we can assign a constant $d=d_{k, p}>0$ such that $\mathcal{F}^{k}$ has the property $P_{\delta, d}^{a}$ at $p$. We have freedom in choosing the value of $d_{k, p}$ but we can choose the largest $d \in(0,1]$ such that $\mathcal{F}^{k}$ has the property $P_{\delta, d}^{a}$ at $p$. Then for fixed $k, d_{k, p}$ is upper semicontinuous in $p$, namely $d_{k, p_{0}} \geq \lim _{p \rightarrow p_{0}} d_{k, p}$.

Lemma 5.2. At any point $p=(x, t) \in \mathcal{F}^{k}$, the principal curvatures of $F_{t}^{k}$ at $x$ satisfy

$$
\Lambda_{\mathcal{F} k}(x, t)<2 C_{\delta, n}^{\prime \prime} / d_{k, p},
$$

provided $k$ is sufficiently large.

Proof. If the lemma is not true, let

$$
\tilde{t}_{k}=\sup \left\{t^{\prime}: \quad \Lambda_{\mathcal{F}^{k}}(p) \leq 2 C_{\delta, n}^{\prime \prime} / d_{k, p}, p=(x, t) \in \mathcal{F}^{k}, \forall x \in F_{t}^{k}, t \leq t^{\prime}\right\} .
$$

For any given $k$, since $d_{k, p}$ is upper semi-continuous in $p$ and $\mathcal{F}^{k}$ is a smooth mean curvature flow, there exists a point $\tilde{p}_{k}=\left(\tilde{x}_{k}, \tilde{t}_{k}\right) \in \mathcal{F}^{k}$ such that

$$
\begin{aligned}
& \Lambda_{\mathcal{F}^{k}}\left(\tilde{p}_{k}\right) \geq 2 C_{\delta, n}^{\prime \prime} / d_{k, \tilde{p}_{k}}, \\
& \Lambda_{\mathcal{F}^{k}}(p) \leq 2 C_{\delta, n}^{\prime \prime} / d_{k, p} \quad \forall p=(x, t) \in \mathcal{F}^{k} \text { with } t<\tilde{t}_{k} .
\end{aligned}
$$

Let $\tilde{\mathcal{F}}^{k}=\mathcal{D}_{\tilde{a}_{k}, \tilde{p}_{k}}\left(\mathcal{F}^{k}\right)$, where $\tilde{a}_{k}=d_{k, \tilde{p}_{k}}^{-1}$ and $\mathcal{D}$ is the dilation in (3.1). Then for any point $p=(x, t) \in \tilde{\mathcal{F}}^{k}$ with $t<0$, the principal curvatures of $\tilde{\mathcal{F}}^{k}$ satisfy $\Lambda_{\tilde{\mathcal{F}}^{k}}(x, t) \leq$ $2 C_{\delta, n}^{\prime \prime} / \tilde{d}_{k, p}$, where $\tilde{d}_{k, p} \in(0,1]$ is the largest constant such that $\tilde{\mathcal{F}}^{k}$ has the property $P_{\delta, \tilde{d}_{k, p}}^{a}$ at $p$. By our definition, $\tilde{d}_{k, p}=1$ at $p=(0,0)$. Hence there is a ball $\mathbb{B}_{\delta}(\tilde{y})$ contained in the interior of $\tilde{F}_{t}^{k}$ for all $t \leq 0$.

Now we apply Lemma 5.1 to $\tilde{F}_{t}^{k}$ with the ball $\mathbb{B}_{r}(0)$ in Lemma 5.1 replaced by $\mathbb{B}_{\delta / 8}(\tilde{y})$. Note that the condition $P_{\delta, d}^{a}$ is weaker than the cone condition in Lemma 5.1. By (5.5) and the curvature pinching estimate (1.1), at any point $p=(x, t) \in \tilde{\mathcal{F}}^{k}$ with $t<0$, the principal curvatures of $\tilde{\mathcal{F}}^{k} \geq-\varepsilon$ with $\varepsilon \rightarrow 0$ as $k \rightarrow \infty$. Hence by Lemma 
5.1, $\tilde{F}_{t}^{k} \cap \mathbb{B}_{R}(0)$ is a Lipschitz continuous radial graph (with respect to $\tilde{y}$ ) when $t<0$. Therefore $\tilde{\mathcal{F}}^{k}$ sub-converges locally smoothly to a limit flow $\tilde{\mathcal{F}}^{\prime}$, which is convex by Lemma 4.1. Hence when $k$ is sufficiently large, $\tilde{F}_{t}^{k}$ satisfies the conditions (a) and (b) above. Sending $t \nearrow 0$ we obtain by (5.2) the curvature estimate $\Lambda_{\tilde{\mathcal{F}}^{k}}(0) \leq C_{\delta, n}^{\prime \prime}$, which is in contradiction with (5.4). $\mathrm{\square}$

From estimate (5.3) and Lemma 5.1, we see that $F_{t}^{k} \cap \mathbb{B}_{R}(0)$ is a Lipschitz continuous radial graph (with respect to some point) for $t \in\left(-R^{2}, 0\right]$. Therefore we obtain the following local smooth convergence.

Corollary 5.1. If a blow-up sequence $\mathcal{F}^{k}$ is semi-collapsing, then the noncollapsing component of $\mathcal{F}^{k}$ sub-converges locally smoothly to a convex limit flow.

REMARK 5.1. To conclude that $\mathcal{F}^{k}$ itself converges locally smoothly, we need to rule out the possibility that $\mathcal{F}^{k}$ contains two or more components, one is the seminoncollapsing component and the others are collapsing (the argument in $\S 4$ implies that a blow-up sequence cannot contain two or more semi-noncollapsing components).

Let $p_{0}=\left(x_{0}, t_{0}\right)$ be a point inside the collapsing component (we need to translate $\mathcal{F}^{k}$ slightly such that the collapsing component of $\mathcal{F}^{k}$ share a common point $p_{0}$ for all $k$ ), and let $\mathbb{B}_{2 r}\left(p_{1}\right)$ be a ball in $U_{0}^{\prime}$ (we assume without loss of generality that $U_{0}^{\prime} \neq \emptyset$ ). Let $\mathcal{C}=\left\{q=t p_{0}+(1-t) p: t \in(0,1), p \in \mathbb{B}_{r}\left(p_{1}\right)\right\}$ be a convex cone with vertex at $p_{0}$. As in the proof of Lemma 4.1, let

$$
\hat{t}_{k}=\sup \left\{s: \mathcal{C} \subset U_{t}^{k} \forall t<s\right\} .
$$

At time $\hat{t}_{k}$, there is a point $\hat{x}_{k} \in \partial \mathcal{C} \cap F_{\hat{t}_{k}}^{k}, \hat{x}_{k} \neq x_{0}$. Let $P_{k}$ be the tangent plane of $F_{\hat{t}_{k}}^{k}$ at $\hat{x}_{k}$. Assume $P_{k} \rightarrow P_{0}$ as $k \rightarrow \infty$. Making the translation $(x, t) \rightarrow\left(x, t-\hat{t}_{k}\right)$, we get a new sequence $\hat{\mathcal{F}}^{k}$. Obviously $\hat{\mathcal{F}}^{k}$ is semi-noncollapsing. By Corollary 5.1, it converges locally smoothly to a convex limit flow $\hat{\mathcal{F}}^{\prime}$. The smooth convergence implies that $P_{0}$ is the tangent plane of $\hat{F}_{0}^{\prime}$ at 0 , where $\hat{F}_{0}^{\prime}$ is the hypersurface of $\hat{\mathcal{F}}^{\prime}$ at time $t=0$. The convexity implies the interior $\hat{U}_{0}^{\prime}$ of $\hat{F}_{0}^{\prime}$ lies on one side of $\hat{P}_{0}$. But on the other hand, the ball $\mathbb{B}_{r}\left(p_{1}\right)$ is tangent to $P_{0}$ and $\mathbb{B}_{2 r}\left(p_{1}\right)$ is contained in $\hat{U}_{0}^{\prime}$, we reach a contradiction.

Therefore we have proved the following theorem. We point out that Corollary 5.1 is sufficient for the treatment in the next section to rule out collapsing.

TheOREM 5.1. Let $\mathcal{F}^{k}$ be a semi-noncollapsing blow-up sequence. Then it converges locally smoothly to a convex limit flow. In particular, $\left\{F_{t}^{\prime}\right\}=\left\{\partial U_{t}^{\prime}\right\}$ is a convex solution to the mean curvature flow.

REMARK 5.2. The arguments above also applies to a collapsing blow-up sequence $\mathcal{F}^{k}$ provided $\mathcal{F}^{k}$ is locally separate. More precisely, let $\mathcal{F}^{k}=\left\{F_{t}^{k}\right\}_{t \in[-\hat{T}, 0)}$ be a blow-up sequence (here we restrict the time $t$ to a fixed interval). Denote

$$
\mathcal{C}_{R}=\left\{x=\left(x^{\prime}, x_{n+1}\right) \in \mathbb{R}^{n+1}:\left|x^{\prime}\right|<R\right\}
$$

a cylinder in $\mathbb{R}^{n+1}$. Suppose for all $t \in[-\hat{T}, 0]$,

$$
F_{t}^{k} \cap \mathcal{C}_{R} \subset\left\{\left|x_{n+1}\right| \leq 10^{-3}\right\},
$$

and $F_{t}^{k} \cap \mathcal{C}_{R}$ consists of two disconnected components,

$$
F_{t}^{k} \cap \mathcal{C}_{R}=G_{1, t}^{k} \cup G_{2, t}^{k},
$$


such that the projection of both $G_{1, t}^{k}$ and $G_{1, t}^{k}$ in the plane $\left\{x_{n+1}=0\right\}$ covers the ball $\mathbb{B}_{R}^{n}(0)$. Then $G_{1, t}^{k}$ divides the ball $\mathbb{B}_{R+t}(0)$ into two separate open sets (we choose the variable radius $R+t$ such that the sets $U_{1, t}^{k}$ and $\mathbb{B}_{k}^{+}$depend continuous on $t$. The continuous dependence is needed in the proof of Lemma 5.2). Let $\mathbb{B}_{k}^{+}$be one of the open sets which contains $U_{t}^{k} \cap \mathbb{B}_{R+t}(0)$, and let $U_{1, t}^{k}=U_{t}^{k} \cup \mathbb{B}_{k}^{+}$. The proof of Lemma 4.1 implies that the limit of $\mathbb{B}_{k}^{+}$is convex. To extend Lemma 5.2 to the current case, we define $d_{k, p}$ using the open set $U_{1, t}^{k}$ (for $p \in G_{1, t}^{k} \cap \mathbb{B}_{R+t}(0)$, for other points on $\mathcal{F}^{k}$, we define $d_{k, p}$ as in Lemma 5.2). Then the argument above implies that $G_{1, t}^{k}$ converges locally smoothly to a convex hypersurface. Similarly $G_{2, t}^{k}$ converges locally smoothly to a convex hypersurface.

6. Semi-noncollapsing. In this section we prove that every blow-up sequence is semi-noncollapsing. The proof consists of two steps, in the first one we show that if every collapsing blow-up sequence is double sheeting, then there is no collapsing blow-up sequence (Lemma 6.2). In the second one we show that every collapsing blow-up sequence must be double sheeting (Lemma 6.4).

First we introduce the notion of double sheeting. We say a blow-up sequence $\mathcal{F}^{k}$ is double sheeting if for any $R>0, \varepsilon>0$, there exists $k_{R, \varepsilon}>1$ such that for all $k \geq k_{R, \varepsilon}, t \in\left(-\varepsilon^{-1},-\varepsilon\right)$,

$$
F_{t}^{k} \cap \mathcal{C}_{R}=G_{f^{k}} \cup G_{g^{k}},
$$

where $G_{f^{k}}$ and $G_{g^{k}}$ are graphs of functions $f^{k}=f^{k}\left(x^{\prime}, t\right)$ and $g^{k}=g^{k}\left(x^{\prime}, t\right)$, in the form

$$
\begin{aligned}
& G_{f^{k}}=\left\{x=\left(x^{\prime}, x_{n+1}\right): x_{n+1}=f^{k}\left(x^{\prime}, t\right),\left|x^{\prime}\right|<R\right\}, \\
& G_{g^{k}}=\left\{x=\left(x^{\prime}, x_{n+1}\right): x_{n+1}=g^{k}\left(x^{\prime}, t\right),\left|x^{\prime}\right|<R\right\},
\end{aligned}
$$

such that

$$
U_{t}^{k} \cap \mathcal{C}_{R}=\left\{g^{k}\left(x^{\prime}, t\right)<x_{n+1}<f^{k}\left(x^{\prime}, t\right)\right\} .
$$

Moreover, for any $\left|x^{\prime}\right|<R,-\varepsilon^{-1}<t<-\varepsilon$, and $k \geq k_{R, \varepsilon}$,

$$
\sum_{i=0}^{4}\left\{\left|D_{x^{\prime}}^{i} f^{k}\left(x^{\prime}, t\right)\right|+\left|D_{x^{\prime}}^{i} g^{k}\left(x^{\prime}, t\right)\right|\right\} \leq \varepsilon .
$$

By the mean convexity of $F_{t}^{k}, f^{k}$ is decreasing, and $g^{k}$ is increasing, in $t$.

Let $\mathcal{F}^{k}=\left\{F_{t}^{k}\right\}$ be a blow-up sequence. For any $x \in \bar{U}_{t}^{k}$ (where $\bar{U}$ is the closure of $U$ ), denote

$$
r_{k, x, t}=\sup \left\{\rho: x \in \overline{\mathbb{B}}_{\rho}(z) \text { and } \mathbb{B}_{\rho}(z) \subset U_{t}^{k} \text { for some } z \in U_{t}^{k}\right\} .
$$

Recall that by our definition in $\S 3$, the origin in space-time is a point in $\mathcal{F}^{k}$. For any given $\tau<0$, if there is a positive constant $c_{0}$ such that

$$
r_{k, 0, \tau} \geq c_{0} \quad \forall k
$$

by Theorem $5.1, \mathcal{F}^{k}$ converges locally smoothly to a convex limit flow. If

$$
r_{k, 0, \tau} \rightarrow 0 \text { as } k \rightarrow \infty
$$


let $\widetilde{\mathcal{F}}^{k}=\mathcal{D}_{a_{k}, p_{k}}\left(\mathcal{F}^{k}\right)$, where $a_{k}=1 / r_{k, 0, \tau}, p_{k}=(0, \tau)$, and $\mathcal{D}$ is the dilation in (3.1). By Theorem 5.1, $\widetilde{\mathcal{F}}^{k}$ converges locally smoothly to a convex limit flow $\widetilde{\mathcal{F}}^{\prime}=\left\{\widetilde{F}_{t}^{\prime}\right\}_{t \in \mathbb{R}^{1}}$.

LEMma 6.1. The limit flow $\widetilde{\mathcal{F}}^{\prime}$ is a pair of parallel planes, namely, $\widetilde{F}_{t}^{\prime}$ is a pair of parallel planes for all $t \in \mathbb{R}^{1}$.

Proof. By (6.4) and the smooth convergence of $\widetilde{\mathcal{F}}^{k}$ to $\widetilde{\mathcal{F}}^{\prime}, \widetilde{U}_{0}^{\prime}$ does not contain a ball $\mathbb{B}_{r}(z)$ such that $r>1$ and $0 \in \overline{\mathbb{B}}_{r}(z)$. Hence $\widetilde{U}_{0}^{\prime}$ cannot be a half space and $\widetilde{F}_{0}^{\prime}$ cannot be a (single) plane.

If the limit flow $\widetilde{\mathcal{F}}^{\prime}$ is not a pair of parallel planes, then the mean curvature of $\widetilde{\mathcal{F}}^{\prime}$ is positive everywhere and it will pass through the origin in finite time. But on the other hand, since $\tau<0$, by the parabolic dilation above, the origin is contained in $U_{t}^{\prime}$ for all $t>0$. This is a contradiction.

Note that by Hamilton's maximum principle for tensors, the second fundamental form of a convex mean curvature flow has constant rank [12]. It implies the mean curvature is positive everywhere.

Therefore if $r_{k, 0, \tau} \rightarrow 0$ for some $\tau<0$, then $\mathcal{F}^{k}$ is a collapsing blow-up sequence, and for any given $t<0, U_{t}^{k}$ does not contain a common ball for all large $k$ (this property will be used in the argument below). Indeed, if $U_{t}^{k}$ (for some $t<0$ ) contains a common ball for a subsequence $k \rightarrow \infty$, by Theorem 5.1, $\mathcal{F}^{k}$ converges locally smoothly to a convex limit flow $\mathcal{F}^{\prime}$. Suppose $\mathcal{F}^{\prime}$ becomes extinct at $T^{\prime}$. By the smooth convergence $\mathcal{F}^{k} \rightarrow \mathcal{F}^{\prime}$ and the convexity of $\mathcal{F}^{\prime}$, it is not hard to show that $\mathcal{F}^{k} \cap \mathbb{B}_{R}(0)$ becomes extinct before $T^{\prime}+\varepsilon_{k}$ with $\varepsilon_{k} \rightarrow 0$ as $k \rightarrow \infty$.

Lemma 6.2. If every collapsing blow-up sequence is double sheeting, then collapsing blow-up sequence does not occur.

Proof. Let $\mathcal{F}=\left\{F_{t}\right\}_{t \in[0, T)}$ be a mean convex flow which develops first time singularity at time $T$. Let $\mathcal{U}_{T}=\mathcal{U} \cap\{t \leq T\}$ (see notation in $\S 3$ ). Regard $\mathcal{U}_{T}$ as a domain in the space-time. For any small $r>0$, and any given point $p=\left(x_{0}, t_{0}\right) \in \partial \mathcal{U}_{T}$ with $t_{0} \geq \frac{1}{2} T$, denote

$$
\begin{aligned}
w_{p}(r) & =\sup \left\{\rho: x_{0} \in \overline{\mathbb{B}}_{\rho}(z) \text { and } \mathbb{B}_{\rho}(z) \subset U_{t_{0}-r^{2}}\right\}, \\
w(r) & =\inf _{p} w_{p}(r) .
\end{aligned}
$$

Since $F_{t}$ is smooth, closed, and mean convex for $t<T$, we have

(i) $w$ is continuous and non-decreasing in $r$;

(ii) $w(r)>0$ for any $r>0$;

(iii) $w(r)$ is attained at some point $\left(x_{r}, t_{r}\right)$.

If there is a collapsing blow-up sequence, we have

$$
\varliminf_{r \rightarrow 0} \frac{w(r)}{r}=0 .
$$

Thus there must be a sequence $r_{i} \rightarrow 0$ such that $\frac{w\left(r_{i}\right)}{r_{i}} \rightarrow 0$ and

$$
\frac{w\left(r_{i}\right)}{r_{i}} \leq 2 \frac{w\left(3 r_{i}\right)}{3 r_{i}} \text {. }
$$

Suppose $w\left(r_{i}\right)$ is attained at $p_{i}=\left(x_{i}, t_{i}\right)$. By definition we have $w_{p_{i}}\left(r_{i}\right)=w\left(r_{i}\right)$ and $w_{p_{i}}\left(3 r_{i}\right) \geq w\left(3 r_{i}\right)$. Hence

$$
\frac{w_{p_{i}}\left(r_{i}\right)}{r_{i}} \leq 2 \frac{w_{p_{i}}\left(3 r_{i}\right)}{3 r_{i}}
$$


Let $\mathcal{F}^{k}=\mathcal{D}_{r_{i}^{-1}, p_{i}}(\mathcal{F})$ be the blow-up sequence of $\mathcal{F}$ at $p_{i}$. Since $\frac{w_{p_{i}}\left(r_{i}\right)}{r_{i}} \rightarrow 0$, $r_{k, 0, \tau} \rightarrow 0$ at $\tau=-1$, where $r_{k, 0, \tau}$ is the number given in (6.4), relative to the blowup sequence $\mathcal{F}^{k}$. Hence $\mathcal{F}^{k}$ is collapsing. Hence by assumption, $\mathcal{F}^{k}$ is double sheeting. Suppose the two sheets are given by (6.2). Denote

$$
\begin{aligned}
& u^{k}(x, t)=\left(f^{k}-g^{k}\right)(x, t), \\
& \hat{u}^{k}(x, t)=u^{k}(x, t) / u^{k}(0,-1),
\end{aligned}
$$

Then $u^{k}$, together with its first and second derivatives, converges locally uniformly to 0 in the parabolic cube $Q_{R}(0,-1)$, for any $R>1$. Note that $f^{k}$ and $g^{k}$ satisfy the parabolic equation (2.2). Hence $u^{k}$ and $\hat{u}^{k}$ satisfy a parabolic equation in $Q_{R}(0,-1)$ which converges as $k \rightarrow \infty$ locally uniformly to the heat equation. Since $\hat{u}^{k}(\cdot, t)$ is decreasing in $t$, the Harnack inequality

$$
\sup _{Q_{R}(0,-1)} \hat{u}^{k} \leq C \inf _{Q_{R}(0,-1)} \hat{u}^{k}
$$

holds, where the constant $C$ is independent of both $k$ and $R$, provided $k$ is sufficiently large such that $\hat{u}^{k}$ is well-defined in $Q_{2 R}(0,-1)$ and satisfies the double sheeting condition. Hence by the regularity of parabolic equation, $\hat{u}^{k}$ converges locally smoothly to a function $\hat{u}$ which is nonincreasing in $t$, and satisfies the heat equation in $\mathbb{R}^{n} \times(-\infty,-1]$. Applying the above Harnack inequality to $\hat{u}-\inf _{\mathbb{R}^{n}} \hat{u}$ in $Q_{R}(0,-1)$ and sending $R \rightarrow \infty$, we concludes that $\hat{u}-\inf \hat{u} \equiv 0$, namely $\hat{u} \equiv \inf \hat{u}$ in $\mathbb{R}^{n}$. Hence $\hat{u}^{k}$ converges locally smoothly to $\hat{u}(0,-1)=1$ in $\mathbb{R}^{n}$. On the other hand, by (6.6) we have $w_{p_{i}}\left(3 r_{i}\right) \geq \frac{3}{2} w_{p_{i}}\left(r_{i}\right)$. Hence

$$
\hat{u}(0,-9)=\lim _{k \rightarrow \infty} \hat{u}^{k}(0,-9) \geq \frac{3}{2} \lim _{k \rightarrow \infty} \hat{u}^{k}(0,-1)=\frac{3}{2},
$$

we reach a contradiction.

The above proof is inspired by the proof of Theorem 9.2 in [21] (pages 688-690). Next we show that every collapsing blow-up sequence must be double sheeting. First we prove

Lemma 6.3. Let $\mathcal{F}^{k}$ be a collapsing blow-up sequence. Then for any $\varepsilon>0$, there exists $k_{\varepsilon}>1$ such that after a rotation of axes,

$$
F_{t}^{k} \cap \mathcal{C}_{\varepsilon^{-1}} \subset\left\{\left|x_{n+1}\right|<\varepsilon\right\}
$$

for all $t>-\varepsilon^{-1}$ and $k>k_{\varepsilon}$.

Proof. For any $m>1$, let

$$
t_{k, m}=\sup \left\{\tau: r_{k, 0, \tau}>1 / m\right\},
$$

where $r_{k, 0, \tau}$ is given in (6.4). Then $t_{k, m} \rightarrow-\infty$. Translating $\mathcal{F}^{k}$ forward in time by $t_{k, m}$, such that $\left(0, t_{k, m}\right)$ becomes the origin in space-time, we get a blow-up sequence $\mathcal{F}_{m}^{k}$ such that $\{0\} \in \mathbb{B}_{1 / m}(z) \subset\left(U_{m}^{k}\right)_{0}$ (the interior of $\mathcal{F}_{m}^{k}$ at $t=0$ ). By Theorem 5.1, $\mathcal{F}_{m}^{k}$ converges locally smoothly to a convex limit flow $\mathcal{F}_{m}^{\prime}$.

From the proof of Lemma $6.1, \mathcal{F}_{m}^{\prime}$ is a pair of parallel planes, namely $\left(F_{m}^{\prime}\right)_{t}$ is a pair of parallel planes for all $t \in \mathbb{R}^{1}$. For if not, the flow $\mathcal{F}_{m}^{\prime}$ would pass through the origin in finite time. But on the other hand, we have $t_{k, m} \rightarrow-\infty$, which means the origin is contained in $\left(U_{m}^{\prime}\right)_{t}$ for all $t>0$. 
By a rotation of the axes, we assume the parallel planes is given by $\left\{x_{n+1}=c_{1}\right\}$ and $\left\{x_{n+1}=c_{2}\right\}$, for some constants $c_{1}, c_{2}$ satisfying $c_{1} \leq 0 \leq c_{2}$ and $c_{2}-c_{1} \leq 5 / \mathrm{m}$. Hence for any given $R$,

$$
\mathcal{C}_{R} \cap F_{t_{k, m}}^{k} \subset\left\{\left|x_{n+1}\right|<10 / m\right\}
$$

provided $k$ is sufficiently large. By the mean convexity, we have

$$
\mathcal{C}_{R} \cap F_{t}^{k} \subset\left\{\left|x_{n+1}\right|<10 / m\right\} \quad \forall t>t_{k, m}
$$

provided $k$ is sufficiently large. We obtain (6.8) by choosing $m=10 / \varepsilon$ and $R=\varepsilon^{-1}$.

For any $m>1, \mathcal{F}_{m}^{k}$ is semi-noncollapsing. The above proof implies that $F_{t}^{k}$ is asymptotically double sheeting when $t \in\left(t_{k, m}, t_{k, 2 m}\right)$, for any sufficiently large $m$. Therefore in the following it suffices to consider the time $t$ in the range $0>t>t_{k, m}$.

For any $\tau<0$, let $\widetilde{\mathcal{F}}^{k}=\mathcal{D}_{a_{k}, p_{k}}\left(\mathcal{F}^{k}\right)$, where $a_{k}=1 / r_{k, 0, \tau}, p_{k}=(0, \tau)$. By Lemma $6.1, \widetilde{\mathcal{F}}^{k}$ converges locally smoothly to a pair of parallel planes, given by $\left\{x_{n+1}=c_{1}\right\}$ and $\left\{x_{n+1}=c_{2}\right\}$. Hence for any $R>0$, when $k$ is sufficiently large, $\widetilde{F}_{t}^{k} \cap \mathcal{C}_{R}$ consists of two separate parts, which are graphs of $\widetilde{f}^{k}$ and $\widetilde{g}^{k}$, given by $x_{n+1}=\widetilde{f}^{k}\left(x^{\prime}, t\right)$ and $x_{n+1}=\widetilde{g}^{k}\left(x^{\prime}, t\right)$, and $\widetilde{f}^{k} \rightarrow c_{1}, \widetilde{g}^{k} \rightarrow c_{2}$ uniformly for $x^{\prime} \in \mathbb{B}_{R}(0)$ and $-R^{2}<t<0$.

Rescaling back we see that for any time $t<0, F_{t}^{k} \cap \mathcal{C}_{r}$ is double sheeting locally for small $r>0$, namely it consists of two separate parts $G_{f^{k}}$ and $G_{g^{k}}$, as given in (6.2). Denote

where

$$
V_{k, \tau, r}=\left\{x \in U_{\tau}^{k}: \quad d_{0, x}\left[U_{\tau}^{k}\right]<r\right\},
$$

$$
d_{x, y}[U]=\inf \{\text { arclength of paths contained in } U \text {, connecting } x \text { to } y\} .
$$

From the above discussion, for $r>0$ small, $\left(\partial V_{k, \tau, r}\right) \cap F_{t}^{k}$ consists of two separate components $\mu_{k, \tau, r}^{+}$and $\mu_{k, \tau, r}^{-}$. Let

$$
\begin{gathered}
\rho_{k, \tau}=\sup \left\{\bar{r}: \text { the two separate parts } \mu_{k, \tau, r}^{+} \text {and } \mu_{k, \tau, r}^{-}\right. \\
\text {keep separate for all } r \in(0, \bar{r})\} .
\end{gathered}
$$

We point out that $\mu_{k, \tau, r}^{+}$and $\mu_{k, \tau, r}^{-}$are the graphs $G_{f^{k}}$ and $G_{g^{k}}$ when $r$ is small, but may not be graph when $r$ is large. Let $y_{k, \tau}$ be the point at which the two parts $\mu_{k, \tau, \rho_{k, \tau}}^{+}$and $\mu_{k, \tau, \rho_{k, \tau}}^{-}$meet.

Lemma 6.4. If $\mathcal{F}^{k}$ is a collapsing blow-up sequence, then it is double sheeting.

Proof. We need to consider three different cases.

Case 1: $\left|y_{k, \tau}\right| \rightarrow \infty$ for all $\tau<0$. In this case, for any given $R>0, \mathcal{C}_{R} \cap F_{t}^{k}$ consists of two separate components for all $t \leq \tau$. By Remark 5.2 and Lemma 6.3, each of the two components converges locally smoothly to the plane $\left\{x_{n+1}=0\right\}$. Hence $\mathcal{F}^{k}$ is double sheeting.

Case 2: $\left|y_{k, \tau}\right| \rightarrow a_{0}>0$ for some $\tau<0$. As in Case $1, \mathcal{C}_{R} \cap F_{t}^{k}$ (for any $R<a_{0}$ ) consists of two separate components which converges locally smoothly to the plane $\left\{x_{n+1}=0\right\}$. Let $\ell_{k, \tau}$ be the shortest path contained in $\bar{U}_{\tau}^{k}$ which connects 0 to $y_{k, \tau}$. Then by definition, the arclength of $\ell_{k, \tau}$ is equal to $\rho_{k, \tau}$. Since $F_{t}^{k}$ is smooth for all $t<0$, there is a point $\hat{y}_{k, \tau} \in \ell_{k, \tau}$ such that

$$
r_{k, \hat{y}_{k, \tau}, \tau}=\frac{1}{10} d_{\hat{y}_{k, \tau}, y_{k, \tau}}\left[U_{\tau}^{k}\right]
$$


Let $\hat{\mathcal{F}}^{k}=\mathcal{D}_{a_{k}, p_{k}}\left(\mathcal{F}^{k}\right)$, where $a_{k}=1 / r_{k, \hat{y}_{k, \tau}, \tau}, p_{k}=\left(\hat{y}_{k, \tau}, \tau\right)$. We get a new blow-up sequence which converges to a convex limit flow $\hat{\mathcal{F}}^{\prime}$ with a nonempty interior. By (6.11), the mean curvature of $\hat{F}_{0}^{\prime}$ is strictly positive at the point $D_{a_{k}, p_{k}}\left(y_{k, \tau}\right)$, i.e. $H \geq C_{0}>0$. Scaling back, we see that the mean curvature of $F_{\tau}^{k}$ at $y_{k, \tau}$ is greater than $C_{0} a_{k} \rightarrow \infty$. Hence

$$
\frac{\partial}{\partial t} \rho_{k, t} \leq-C a_{k} \rightarrow-\infty
$$

as $k \rightarrow \infty$. By Lemma 6.5 below, $\rho_{k, t} \leq 4\left|y_{k, t}\right|$. Hence $\rho_{k, t} \rightarrow 0$ in very short time, which means the blow-up sequence $\mathcal{F}^{k}$ passes through the origin in very short time, and so the origin $0 \notin F_{0}^{k}$, we reach a contradiction.

Case 3: If there exists a $\tau<0$ such that $\left|y_{k, \tau}\right| \rightarrow 0$, we make a parabolic dilation such that $\left|y_{k, \tau}\right|=1$. The new blow-up sequence is still collapsing and so we are in the same situation as in Case 2, which implies the blow-up sequence $\mathcal{F}^{k}$ passes through the origin immediately.

By our choice of the point $y_{k, \tau}$, there are at least $n-1$ vanishing principal curvatures of $\hat{F}_{0}^{\prime}$ (the hypersurface at $t=0$ of the limit flow $\hat{\mathcal{F}}^{\prime}$ in Case 2) at the limit point of $D_{a_{k}, p_{k}}\left(y_{k, \tau}\right)$. Therefore by the constant rank of the second fundamental form of $\hat{\mathcal{F}}^{\prime}$ [12], the limit flow $\hat{\mathcal{F}}^{\prime}$ is the product of $\mathbb{R}^{n-1}$ with a convex solution to the curvature shortening flow. By (6.11), $C_{0}$ is an absolute constant.

LEMma 6.5. Let $\rho_{k, \tau}$ and $y_{k, \tau}$ be as above. If $\left|y_{k, \tau}\right|$ is uniformly bounded, then we have $\rho_{k, \tau} \leq 4\left|y_{k, \tau}\right|$.

Proof. Let $\ell_{k, \tau}$ be the shortest path in $U_{\tau}^{k}$ connecting 0 to $y_{k, \tau}$. For a sequence of points $z_{k} \in \ell_{k, \tau}$, let $r_{k}=r_{k, z_{k}, \tau}$ be given in (6.4) and let $d_{k}=d_{z_{k}, y_{k, \tau}}$ be the arelength of $\ell_{k, \tau}$ from $z_{k}$ to $y_{k, \tau}$.

Let $z_{0}=0$, we choose a sequence of points $z_{m}=z_{m, k} \in \ell_{k, \tau}$ for $m=1,2, \cdots$ such that the angle between $\xi\left(z_{m}\right)$ and $\xi\left(z_{m-1}\right)$ is equal to $\frac{\pi}{6}$, and for any point $z \in \ell_{k, \tau}$ between $z_{m-1}$ and $z_{m}$, the angle between $\xi(z)$ and $\xi\left(z_{m-1}\right)$ is less than or equal to $\frac{\pi}{6}$, where $\xi(z)$ denote the tangent vector of $\ell_{k, \tau}$. If there is no point $z \in \ell_{k, \tau}$ such that the angle between $\xi(z)$ and $\xi\left(z_{0}\right) \geq \frac{\pi}{6}$, Lemma 6.5 is obvious.

Claim. $d_{z_{m}, z_{m+1}} \leq \frac{1}{4} d_{z_{m-1}, z_{m}}$ uniformly in $m$, provided $k$ is sufficiently large.

Indeed, if there is a sequence of $m_{k}$ such that $d_{z_{m_{k}, k}, z_{m_{k}+1, k}} \geq \frac{1}{4} d_{z_{m_{k}, k}, z_{m_{k}-1, k}}$ (recall that $z_{m}=z_{m, k}$ ), by a parabolic dilation, we may assume that $d_{z_{m_{k}, k}, z_{m_{k}-1, k}}=$ 1. If $\mathcal{F}^{k}$ is collapsing after the parabolic dilation, then $\mathcal{F}^{k} \cap \mathbb{B}_{1 / 8}\left(z_{m_{k}, k}\right)$ consists of two separate components and both converges smoothly to a hyperplane (here $\mathbb{B}_{1 / 8}$ is the ball after the dilation). If $\mathcal{F}^{k}$ is semi-noncollapsing after the parabolic dilation, by Theorem 5.1 it converges locally smoothly to a convex solution. In both case we reach a contradiction, as $\ell_{k, \tau}$ is the shortest path connecting $z_{m-1}$ to $z_{m+1}$ but $\operatorname{osc}_{z \in \ell_{z_{m-1}, z_{m+1}}} \xi(z) \geq \frac{\pi}{6}$, where $\ell_{z_{m-1}, z_{m+1}}$ is the part of $\ell_{k, \tau}$ between $z_{m-1}$ and $z_{m+1}$. Hence we obtain

$$
\rho_{k, \tau}=\sum_{m \geq 1} d_{z_{m-1}, z_{m}}\left[U_{\tau}^{k}\right]<2 d_{z_{0}, z_{1}}\left[U_{\tau}^{k}\right] .
$$

Note that for any $R<\left|y_{k, \tau}\right|, \mathcal{F}^{k} \cap \mathbb{B}_{1 / 8}(0)$ consists of two separate components, and each converges smoothly to a hyperplane. Hence we have $\left|y_{k, \tau}\right| \geq \frac{1}{2} d_{z_{0}, z_{1}}$.

We have thus proved that every blow-up sequence is semi-noncollapsing. 
Corollary 6.1. A limit flow cannot be a pair of parallel planes. Moreover, the grim reaper is not a limit flow.

Proof. If a blow-up sequence $\mathcal{F}^{k}$ converges to a multiplicity two plane, then $\mathcal{F}^{k}$ is collapsing and must be double sheeting, which was ruled out in Lemma 6.2. If $\mathcal{F}^{k}$ converges to a pair of parallel planes, we can choose a sequence $a_{k} \rightarrow 0$ such that $\mathcal{D}_{a_{k}, 0}\left(\mathcal{F}^{k}\right)$ is a collapsing blow-up sequence. But any blow-up sequence is seminoncollapsing, we also get a contradiction.

If the grim reaper is a limit flow, then by a proper translation, there is a limit flow which is a pair of parallel planes.

7. Proof of Theorems 1.1-1.3. Theorem 1.1 follows from Theorem 5.1 and the semi-noncollapsing in $\S 6$.

Proof of Theorem 1.2. If there is a sequence of points $p_{k}=\left(x_{k}, t_{k}\right)$ such that

$$
r_{x_{k}, t_{k}} H\left(x_{k}, t_{k}\right)=\delta_{k} \rightarrow 0,
$$

where $r_{x, t}$ is defined in (3.2), we consider the blow-up sequence $\mathcal{F}^{k}=\mathcal{D}_{a_{k}, p_{k}}(\mathcal{F})$, where $a_{k}=1 / r_{x_{k}, t_{k}}$. By Theorem $5.1, \mathcal{F}^{k}$ converges locally smoothly to a convex limit flow $\mathcal{F}^{\prime}$. By (7.1), the mean curvature of $F_{0}^{k}$ at 0 is equal to $\delta_{k}$. Hence the mean curvature of $F_{0}^{\prime}$ vanishes at the origin, which implies $F_{0}^{\prime}$ is a single hyperplane or a pair of parallel planes (by the constant rank of the second fundamental form). The former case is ruled out by our definition of $r_{x, t}$ in (3.2), as it implies that the unit sphere is the largest tangent sphere of $F_{0}^{\prime}$ at the origin. The latter case was ruled out in Corollary 6.1. Hence there is a $\delta>0$ such that $r_{x, t} H(x, t) \geq \delta$ for all point $(x, t) \in \mathcal{F}$.

Proof of Theorem 1.3. If Theorem 1.3 is not true, there is a sequence of normalized limit flows $\mathcal{F}^{\prime k}$ such that $r_{k} \rightarrow 0$, where as in (3.2),

$$
r_{k}=\sup \left\{\rho: \mathbb{B}_{\rho}(z) \subset U_{0}^{\prime k} \text { and }|z-x|=\rho\right\}
$$

is the radius of the largest ball which is contained in $U_{0}^{\prime k}$ and tangential to $F_{0}^{\prime k}$ at 0 .

Regard $\mathcal{F}^{\prime k}$ as a hypersurface in the spacetime $\mathbb{R}^{n+1} \times \mathbb{R}^{1}$, then it is a graph of a function $u_{k}$,

$$
x_{n+2}=u_{k}\left(x_{1}, \cdots, x_{n+1}\right),
$$

where $x_{n+2}=-t$, and $u_{k}$ satisfies the equation

$$
\sum_{i, j=1}^{n+1}\left(\delta_{i j}-\frac{u_{i} u_{j}}{|D u|^{2}}\right) u_{i j}=1 .
$$

Since $\mathcal{F}^{\prime k}$ is normalized, the mean curvature of $F_{0}^{\prime k}=\left\{u_{k}=0\right\}$ is equal to 1 at the origin, namely

$$
\left|D u_{k}(0)\right|=1 .
$$

By Proposition 4.1 [20], $u_{k}$ is a convex function, and by (1.5) [20], we have the estimate

$$
u_{k}(x) \leq C\left(1+|x|^{2}\right)
$$

for some constant $C$ depending only on $n$. Hence $u_{k}$ converges along a subsequence to a convex function $u$, whose level set is still a solution to the mean curvature flow. 
Since $r_{k} \rightarrow 0$ and $\mathcal{F}^{\prime k}$ is convex, the volume of the convex sets $\left\{u_{k}<0\right\} \cap \mathbb{B}_{R}(0)$ converges to zero for any given $R>0$, for otherwise $\mathcal{F}^{\prime k}$ is MCF of graph with uniformly bounded gradient near the origin. Hence we have

$$
u(0)=0, \quad u \geq 0 .
$$

Recall that $\left|D u_{k}(0)\right|=1$ and $u_{k}$ is convex, by a rotation of coordinates we assume that $\left\{x_{n+2}=x_{1}\right\}$ is a supporting plane of $u_{k}$ at 0 , namely $u_{k}(x) \geq x_{1}$ for any $x \in \mathbb{R}^{n+1}$. Hence we also have

$$
u(x) \geq x_{1} \quad \forall x \in \mathbb{R}^{n+1} .
$$

By Lemma 2.6 of [20], the set $\{u=0\}$ is either a single point, or a linear subspace of $\mathbb{R}^{n+1}$.

In the latter case, we may choose the axes such that $\{u=0\}$ is the subspace spanned by the $x_{k+1}, \cdots, x_{n+1}$-axes. Then by convexity, $u$ is independent of the variable $x_{k+1}, \cdots, x_{n+1}$, namely $u(x)=u\left(x_{1}, \cdots, x_{k}\right)$. By restricting $u$ to the subspace $\mathbb{R}^{k}$ spanned by the $x_{1}, \cdots, x_{k}$-axes, it reduces to the former case, namely the set $\{u=0\}$ is a single point.

Since the level set $F_{t}:=\{u=-t\}$ is a convex solution to the mean curvature flow, by Huisken [8], it converges to a round point. Hence we have $u(x)=O\left(|x|^{2}\right)$, which is in contradiction with the estimate $u(x) \geq x_{1}$. Hence $r_{k}$ has a positive lower bound. This completes the proof.

Proof of Corollary 1.3. Assume to the contrary that (1.2) is not true, then there exists $\delta_{k} \rightarrow 0$, and two sequences of points $p_{k}$ and $q_{k}$, where $q_{k} \in Q_{R}\left(p_{k}\right)$, such that $H\left(p_{k}\right)=\delta_{k}^{-1}$ and $H\left(q_{k}\right) \geq \delta_{k}^{-2}$. Let $\mathcal{F}^{k}=\mathcal{D}_{\delta_{k}^{-1}, p_{k}}(\mathcal{F})$ be the normalized blow-up sequence at $p_{k}$. Then $\mathcal{F}^{k} \cap \mathbb{B}_{2 R}(0)$ converges smoothly to a convex hypersurface. Hence $H_{\mathcal{F}^{k}}\left(\hat{q}_{k}\right)$ is uniformly bounded, where $H_{\mathcal{F}^{k}}\left(\hat{q}_{k}\right)$ is the mean curvature of $\mathcal{F}^{k}$ at $\hat{q}_{k}=\mathcal{D}_{\delta_{k}^{-1}, p_{k}}\left(q_{k}\right)$. But by assumption we have $H_{\mathcal{F}^{k}}\left(\hat{q}_{k}\right) \geq \delta_{k}^{-1}$. We reach a contradiction, as $\mathcal{F}^{k}$ converges locally smoothly.

Similarly one can prove the second inequality of (1.2).

8. Tangent flow. First we state the following result, which is due to Huisken [10], see also Ilmanen [15], and White [22].

Proposition 8.1. A tangent flow to a mean convex flow must be a shrinking cylinder, namely $\mathcal{F}^{\prime}=S_{r}^{m} \times \mathbb{R}^{n-m}$ for $1 \leq m \leq n$; or a hyperplane $\mathcal{F}^{\prime}=\mathbb{R}^{n}$, where $S_{r}^{m}(m \geq 1)$ denotes the shrinking sphere to the $M C F$ in $\mathbb{R}^{m+1}$.

From Theorems 1.1 and 1.2, we also have the follow result, which means that the blow-up solution at a fixed first time singular point is a shrinking sphere or cylinder.

COROllary 8.1. A tangent flow at a (first time) singular point cannot be a hyperplane.

Proof. Let $\left(x_{0}, t_{0}\right)$ be a first time singular point. For any $\tau>0$ small, let $x_{\tau} \in$ $F_{t_{0}-\tau}$ be the point closest to $x_{0}$. It suffices to prove that

$$
c_{1} \tau^{1 / 2} \leq r_{\tau} \leq c_{2} \tau^{1 / 2}
$$

where $r_{\tau}=\operatorname{dist}\left(x_{0}, F_{t_{0}-\tau}\right)$.

For the first inequality, consider the normalized blow-up sequence $\mathcal{F}^{\tau}$ at $\left(x_{\tau}, t_{-} \tau\right)$, which converges along a subsequence locally smoothly to a convex limit flow $\mathcal{F}^{\prime}$. By 
the $\kappa$-noncollapsing and since $\mathcal{F}^{\tau}$ is normalized blow-up sequence, there is a sphere of radius $\kappa$, tangent to $F_{0}^{\prime}$ at the origin, contained in $U_{0}^{\prime}$. Hence the limit flow $\mathcal{F}^{\prime}=\left\{F_{t}^{\prime}\right\}$ does not develop singularity for $t \in\left(0, c_{0}\right)\left(c_{0}=\left(\frac{\kappa}{2 n}\right)^{2}\right)$, which is equivalent by the scaling (3.1) to that $H\left(x_{\tau}, t_{0}-\tau\right) \tau^{1 / 2} \geq c_{0}$ (here $H$ is the mean curvature of $\mathcal{F})$. Hence the velocity at $x_{\tau}$ of the MCF is greater than $c_{0} \tau^{-1 / 2}$, which implies that $\frac{d}{d \tau} r_{\tau} \geq c_{0} \tau^{-1 / 2}$. Hence the distance $\left|x_{\tau}-x_{0}\right| \geq c_{0} \tau^{1 / 2}$. We obtain the first inequality.

To prove the second inequality, we consider the sphere $\partial \mathbb{B}_{r_{\tau}}\left(x_{0}\right)$ at time $t_{0}-\tau$, at any given $\tau$. If $r_{\tau}>\sqrt{2 n \tau}$, the above sphere evolving by the MCF will not shrink to the point $x_{0}$ at time $t_{0}$, and so $\left(x_{0}, t_{0}\right)$ cannot be a singular point of the MCF $\mathcal{F}$. $\mathbf{D}$

\section{REFERENCES}

[1] K. Brakke, The motion of a surface by its mean curvature, Princeton Univ. Press, 1978.

[2] Y.G. Chen, Y. Giga And S. Goto, Uniqueness and existence of viscosity solutions of generalized mean curvature flow equation, J. Diff. Geom., 33 (1991), pp. 749-786.

[3] K. ECKER, Regularity theory for mean curvature flow, Birkhauser, Boston, 2004.

[4] K. Ecker AND G. Huisken, Interior estimates for hypersurfaces moving by mean curvature, Invent. Math., 105 (1991), pp. 547-569.

[5] L.C. Evans and J. Spruck, Motion of level sets by mean curvature, I, J. Diff. Geom., 33 (1991), pp. 635-681; II, Trans. Amer. Math. Soc., 330 (1992), pp. 321-332; III, J. Geom. Anal., 2 (1992), pp. 121-150; IV, J. Geom. Anal., 5 (1995), pp. 77-114.

[6] R. Hamilton, The formation of singularities in the Ricci flow, Surveys in differential geometry, Vol. II, pp. 7-136, International Press, 1995.

[7] R. Hamilton, Harnack estimates for the mean curvature flow, J. Differential Geom., 41 (1995), pp. $215-226$.

[8] G. Huisken, Flow by mean curvature of convex surfaces into spheres, J. Diff. Geom., 20 (1984), pp. 237-266.

[9] G. Huisken, Asymptotic behaviour for singularities of the mean curvature flow, J. Diff. Geom. 31 (1990), 285-299.

[10] G. Huisken, Local and global behaviour of hypersurfaces moving by mean curvature, Proc. Symp. Pure Math., 54 (1993), pp. 175-191.

[11] G. Huisken And C. Sinestrari, Mean curvature flow singularities for mean convex surfaces, Calc. Var. PDE, 8 (1999), pp. 1-14.

[12] G. Huisken And C. Sinestrari, Convexity estimates for mean curvature flow and singularities for mean convex surfaces, Acta Math., 183 (1999), pp. 45-70.

[13] G. Huisken and C. Sinestrari, Mean curvature flow with serguries, Inventiones Mathematicae, to appear.

[14] T. Ilmanen, Elliptic regularization and partial regularity for motion by mean curvature, Mem. Amer. Math. Soc., 108:520 (1994).

[15] T. Ilmanen, Singularities of mean curvature flow of surfaces, preprint, 1995.

[16] G. Lieberman, Second order parabolic differential equations, World Scientific, Singapore, 1996.

[17] G. Perelman, The entropy formula for the Ricci flow and its geometric applications, arXiv:math/0211159.

[18] G. Perelman, Ricci flow with surgery on three manifolds, arXiv:math/0303109.

[19] X.-J. WANG, Interior gradient estimates for mean curvature equations, Math. Z., 228 (1998), pp. $73-81$.

[20] X.-J. WANG, Convex solutions to the mean curvature flow, arXiv:math.DG/0404326.

$[21]$ B. White, The size of the singular set in mean curvature flow of mean-convex sets, J. Amer. Math. Soc., 13 (2000), pp. 665-695.

[22] B. White, The nature of singularities in mean curvature flow of mean-convex sets, J. Amer. Math. Soc., 16 (2003), pp. 123-138.

[23] X.-P. ZHU, Lectures on mean curvature flows, International Press, 2002. 
